\title{
BENDING OF AN INCOMPLETE ANNULAR PLATE AND RELATED PROBLEMS
}

J. R. BARBER The University of Newcastle upon Tyne. Member of the Institution

Closed-form solutions and stress-concentration data are obtained for the problem of a sector of an annular plate subjected to moments and transverse forces on its radial edges. Closed-form solutions are also given for a semi-infinite plate or a circular plate subjected to a system of concentrated forces and/or moments at the edge.

\section{INTRODUCTION}

The usefulness of theoretical research results in design depends to a large extent upon their generality and simplicity. For this reason, closed-form solutions are presented in this paper for a number of plate bending problems, these being:

(1) a sector cut from an annular plate, loaded along its straight edges; and

(2) a semi-infinite plate or a circular plate subjected to concentrated moments or forces at one or more points on the edge.

These problems could also be solved by series or complex variable methods, but these would, in general, give Fourier-series solutions.

\subsection{Notation and sign conventions.}

The notation and sign conventions used for components of moment, $M$, and shear force, $Q$, are adapted from those of Mansfield (1) $\dagger$ and are illustrated in Fig. 1. Displacement, $u$, and applied lateral pressure, $p$, are taken as positive in the direction of the $z$-axis.

With these conventions, the flexure of the plate is governed by the equation

$$
\Delta^{4} u=p / D
$$

whilst the moments and shear forces are given by

$$
\begin{aligned}
& M_{r r}=-D\left[\frac{\partial^{2} u}{\partial r^{2}}+v\left(\frac{1}{r} \frac{\partial u}{\partial r}+\frac{1}{r^{2}} \frac{\partial^{2} u}{\partial \theta^{2}}\right)\right] \\
& M_{\theta \theta}=-D\left[\frac{1}{r} \frac{\partial u}{\partial r}+\frac{1}{r^{2}} \frac{\partial^{2} u}{\partial \theta^{2}}+v \frac{\partial^{2} u}{\partial r^{2}}\right] \\
& M_{r \theta}=-D(1-v) \frac{\partial}{\partial r}\left(\frac{1}{r} \frac{\partial u}{\partial \theta}\right) \\
& Q_{r}=-D \frac{\partial}{\partial r} \Delta^{2} u \quad Q_{\theta}=-\frac{D}{r} \frac{\partial}{\partial \theta} \Delta^{2} u
\end{aligned}
$$

(see (1), equations $3.5,3.6)$ where

$$
D=\frac{E t^{3}}{12\left(1-v^{2}\right)}
$$
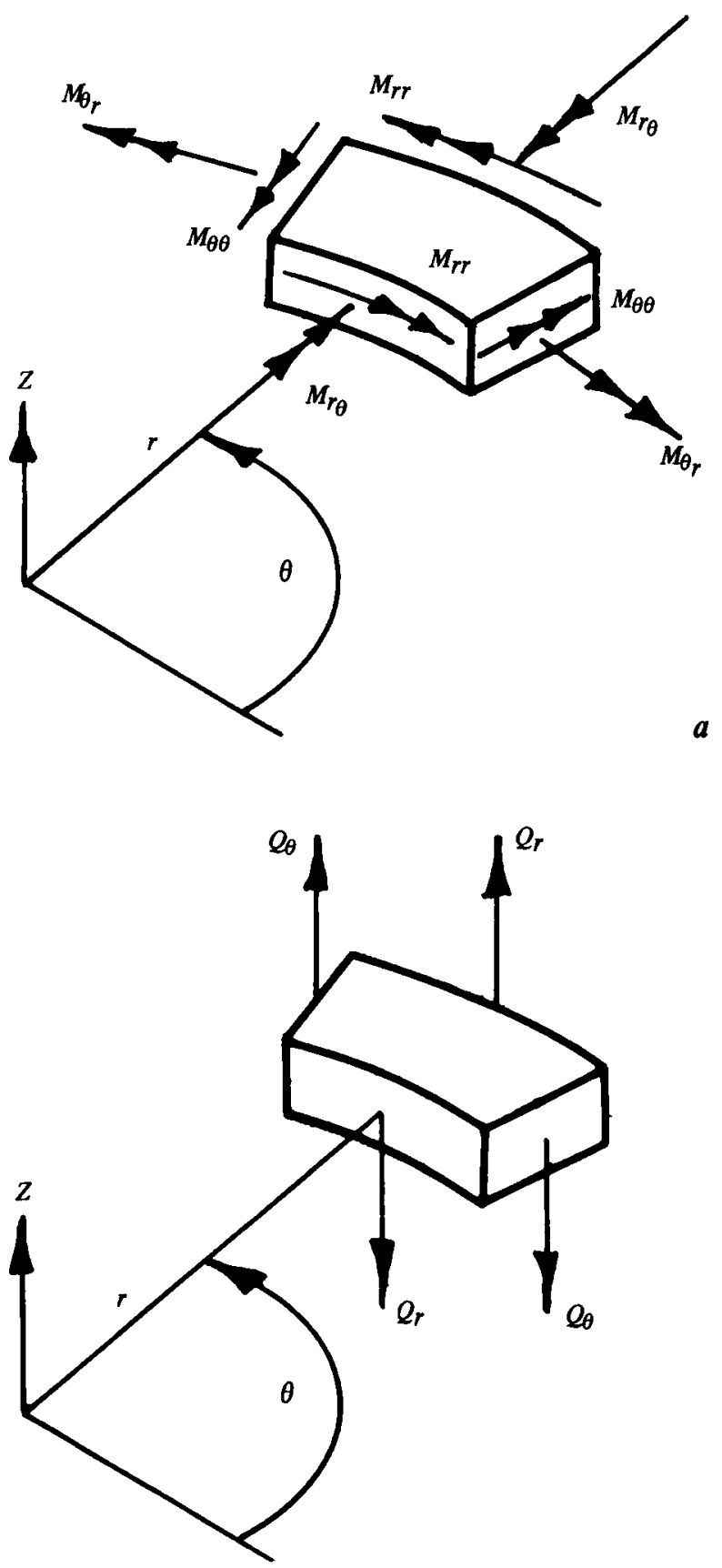

The MS. of this paper was received at the Institution on 17th July 1978 and accepted for publication on 7th February 1979.

+ References are given in the Appendix.

Fig. 1 
$t$ is the thickness of the plate, and $E, v$ are Young's modulus and Poisson's ratio, respectively, for the material.

We also note that the condition for a free edge is At $r=$ constant

$$
M_{r r}=0, Q_{r}+\frac{1}{r} \frac{\partial M_{r \theta}}{\partial \theta}=0
$$

At $\theta=$ constant

$$
M_{\theta \theta}=0, Q_{\theta}+\frac{\partial M_{r \theta}}{\partial r}=0
$$

These are the well-known Kirchhoff relations, which are discussed, for example, in (1), page 18.

\section{THE aNNULAR PLATE IN BENDING}

We consider the problem of the annular plate $a \leqslant r \leqslant b$, $0 \leqslant \theta \leqslant \pi / 2$, built-in at the edge $\theta=\pi / 2$ and loaded by a bending moment, $M_{0}$, along $\theta=0$, as shown in Fig. $2 a$.

The plate is deformed by edge loading only and hence the displacement will be a bi-harmonic function. The statics of the problem suggest that the moments and shear forces should vary with $\cos \theta$ and $\sin \theta$, but if the general bi-harmonic function of this form

$$
u=\left(c_{1} r^{3}+c_{2} r+c_{3} r^{-1}+c_{4} r \log r\right) \sin \theta
$$

is used, the application of the two free-edge conditions at $r=a, r=b$ reduces the solution to the trivial case of zero stress. However, the further bi-harmonic term, $c_{5} r \theta \sin \theta$, also gives force components of the required form and its inclusion permits the solution of certain problems in which a moment is transmitted through the plate.

Substituting

$$
u=\left(c_{1} r^{3}+c_{2} r+c_{3} r^{-1}+c_{4} r \log r\right) \cos \theta+c_{5} r \theta \sin \theta
$$

into equations (2), (3) and (4), we find

$$
\begin{aligned}
& M_{r r}=-D\left[2(3+v) c_{1} r+2(1-v) c_{3} r^{-3}\right. \\
& \left.+(1+v) c_{4} r^{-1}+2 v c_{5} r^{-1}\right] \cos \theta \\
& Q_{r}+\frac{1}{r} \frac{\partial M_{r \theta}}{\partial \theta}=-D\left[2(3+v) c_{1}+2(1-v) c_{3} r^{-4}\right. \\
& \left.-(3-v) c_{4} r^{-2}-2 c_{5} r^{-2}\right]
\end{aligned}
$$

Hence, if we choose $c_{4}, c_{5}$ such that

$$
(1+v) c_{4}+2 v c_{5}=-(3-v) c_{4}-2 c_{5}
$$

that is,

$$
c_{4}=-c_{5}(1+v) / 2
$$

we have

$$
M_{r r}=-r\left(Q_{r}+\frac{1}{r} \frac{\partial M_{r \theta}}{\partial \theta}\right)
$$

for all $r, \theta$, and hence there will be two values of $r$ which satisfy the conditions for a free edge.

It is now a straightforward procedure to choose $c_{1}, \ldots, c_{5}$ to make the surfaces $r=a, r=b$ stress-free and the total moment along $\theta=0$ :

$$
\int_{a}^{b} M_{\theta \theta}(\theta=0) \mathrm{d} r=M_{0}
$$
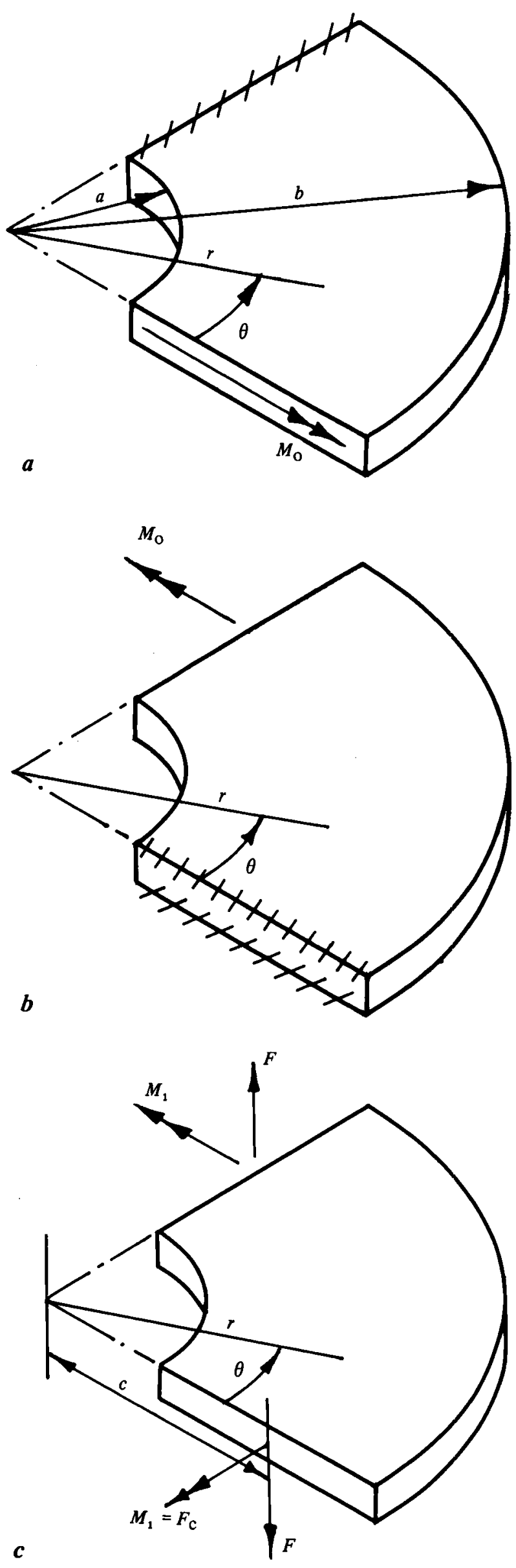

Fig. 2 
The final solution is

$$
\begin{aligned}
& u=\frac{M_{0}}{D N}\left\{\frac{(3+v) r[(1+v) \log r \cos \theta-2 \theta \sin \theta]}{(1-v)}\right. \\
& \left.-\frac{\left[(3+v) a^{2} b^{2}-(1-v) r^{4}\right] \cos \theta}{2\left(a^{2}+b^{2}\right) r}\right\}+c_{2} r \cos \theta \\
& M_{r r}=\frac{M_{0}(3+v)(1-v) \cos \theta}{N r}\left\{\frac{\left(a^{2} b^{2}+r^{4}\right)}{\left(a^{2}+b^{2}\right) r^{2}}-1\right\} \\
& M_{\theta \theta}=\frac{M_{0} \cos \theta}{N r}\left\{(3+v)^{2}+\frac{(1-v)}{\left(a^{2}+b^{2}\right) r^{2}}\left[(1+3 v) r^{4}\right.\right. \\
& \left.\left.-(3+v) a^{2} b^{2}\right]\right\} \\
& M_{r \theta}=\frac{M_{0} \sin \theta}{N r}\left\{(3+v)(1+v)+\frac{(1-v)}{\left(a^{2}+b^{2}\right) r^{2}}\left[(3+v) a^{2} b^{2}\right.\right. \\
& \left.\left.-(1-v) r^{4}\right]\right\} \\
& Q_{r}=-\frac{2 M_{0} \cos \theta}{N}\left\{\frac{(3+v)}{r^{2}}-\frac{2(1-v)}{\left(a^{2}+b^{2}\right)}\right\} \\
& Q_{\theta}=-\frac{2 M_{0} \sin \theta}{N}\left\{\frac{(3+v)}{r^{2}}+\frac{2(1-v)}{\left(a^{2}+b^{2}\right)}\right\}
\end{aligned}
$$

where

$$
N=(3+v)^{2} \log (b / a)-(1-v)^{2}\left\{\frac{b^{2}-a^{2}}{b^{2}+a^{2}}\right\}
$$

It should be noted that equation (5) contains only one more constant than is required to satisfy the two freeedge conditions, and hence the boundary conditions on the straight edges can only be satisfied in terms of force and moment resultants, as in equation (10). This limitation is reflected in the fact that the built-in boundary condition $(\partial u / \partial \theta=0)$ is not satisfied along $\theta=\pi / 2$.

A best approximation for the displacement, $u$, can be achieved by choosing the rigid-body rotation:

$$
\begin{aligned}
& c_{2}=\frac{M_{0}}{D N}\left[\frac{2(2+v) a b}{3\left(a^{2}+b^{2}\right)}-\frac{7(1-v)}{6}\right. \\
& \left.-\frac{(3+v)(1+v)(b \log \mathrm{b}-a \log a)}{(1-v)(b-a)}\right]
\end{aligned}
$$

This has the effect of making the mean value of the slope $(1 / r . \partial u / \partial \theta)$ zero on the edge $a \leqslant r \leqslant b, \theta=\pi / 2$. The value of $c_{2}$, of course, has no effect on the force components, equations (12)-(16). If the edge $\theta=\pi / 2$ is truly built-in (i.e. the slope is zero at all points), some local bending moments, $M_{\theta \theta}$, will be developed, but these must be self-equilibrating and are unlikely to disturb seriously the stress field, following St Venant's principle.

With a similar proviso, the same solution can be used for the problem illustrated in Fig. $2 b$ by regarding the edge $\theta=\pi / 2$ as loaded by a twisting moment, $M_{0}$, whilst $\theta=0$ is built-in.

For design purposes, it is convenient to compare the above solution with a simpler treatment of the same problem. If the ratio $a / b$ is close to unity, the conditions are similar to those in a rectangular bar in bending and torsion; that is, the bending and twisting moments, $M_{\theta \theta}$, are uniform at any radial cross-section.
As $a / b$ is reduced, stress concentrations in these quantities are developed at $r=a$. These are conveniently defined as

$$
\begin{aligned}
& K_{1}=\frac{2(b-a) M_{r \theta}(a, \pi / 2)}{M_{0}} \\
& K_{2}=\frac{(b-a) M_{\theta \theta}(a, 0)}{M_{0}}
\end{aligned}
$$

and are shown in Fig. 3. (Note that, for a rectangular bar in torsion, exactly half the twisting moment is carried by horizontal shear stresses, the remainder being carried by vertical shear stresses near the edges. Hence, a factor of two arises in equation (18).

\section{THE ANNULAR PLATE IN SHEAR}

For completeness, we also give the solution for the annular plate transmitting a shear force, as shown in Fig. $2 c$. If the line of action of the resultant force on any section coincides with the $z$-axis (i.e. $M_{1}=c F$ ), the displacement is given by

$$
u=\frac{F a^{2} b^{2} \theta}{(1-v) D\left(b^{2}-a^{2}\right)}
$$

All the force components are zero, except

$$
M_{r \theta}=\frac{F a^{2} b^{2}}{\left(b^{2}-a^{2}\right) r^{2}}
$$

When $a / b$ is close to unity, $M_{r \theta}$ is uniform across the section, as in the previous problem, and has the value $F(a+b) / 4(b-a)$. We can therefore define a stress concentration factor,

$$
K_{3}=\frac{4(b-a) M_{r \theta}(a, \theta)}{(b+a) F}=\frac{4 b^{2}}{(a+b)^{2}}
$$

which is shown in Fig. 3.

The solution for the case in which the shear force has a more general line of action can be obtained by superposing this and the previous solution.

\section{APPLICATIONS}

More general types of loading may be treated by superposing the above results.

The particular application for which these solutions were developed is that of a large plate supported along part of one edge, the discontinuity being relieved by a small radius, $a$, as shown in Fig. 4.

If the applied load is not too close to the support, an estimate of the stress concentration at D can be obtained by treating the section $A B C D$ as an annular plate with appropriate edge loading at $\mathrm{CB}$.

The same solutions can be used for an incomplete annular plate of any subtended angle, but they cannot be used for the complete ring, since there are discontinuities of slope and displacement at $\theta=2 \pi$. However, the same stress distributions could occur in a complete ring as residual stresses and could be artificially developed (as dislocations) by welding-up rings with appropriate discontinuities. 


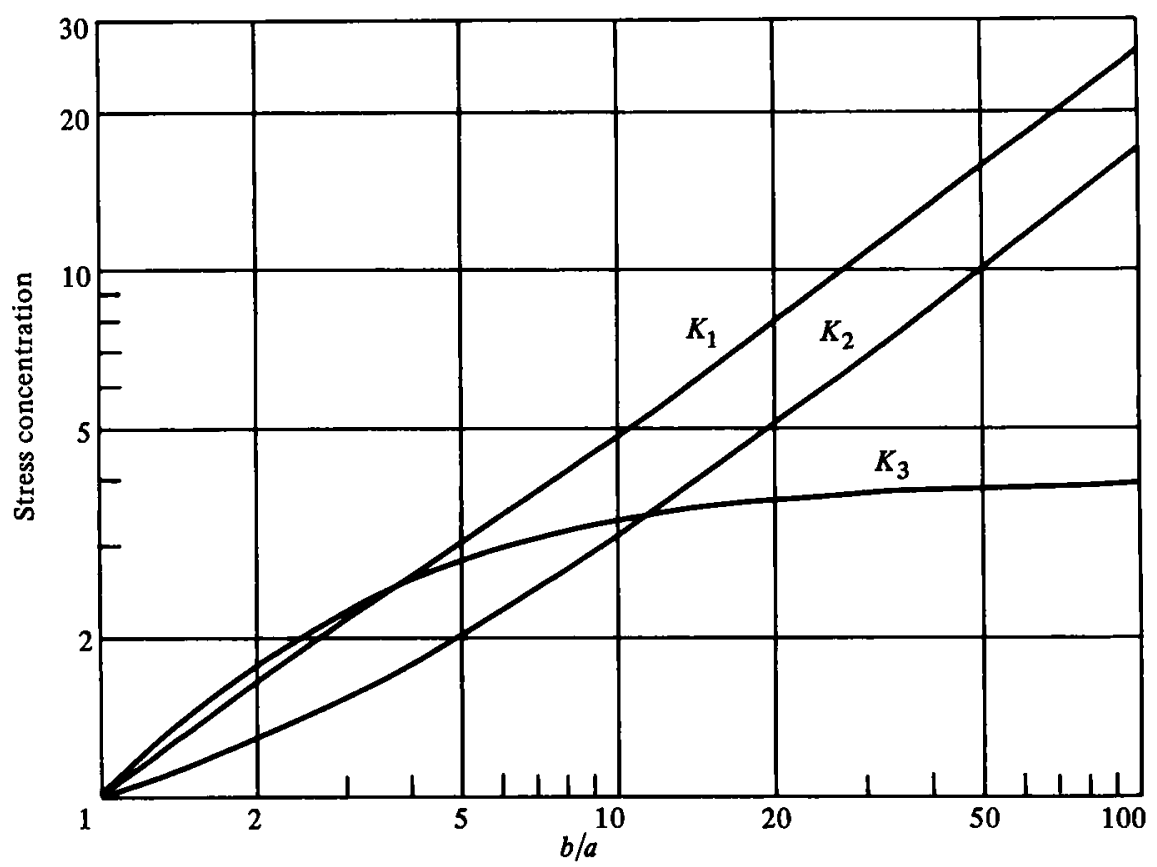

Fig. 3

The same solution could also be used for the tension or bending of a close-coiled spring of rectangular crosssection, the thickness of the plate being in the direction of the axis of the spring.

\section{BENDING OF THE SEMI-INFINITE PLATE BY AN EDGE MOMENT}

The last two terms in equation (5) can be used to develop the solution for the semi-infinite plate, $-\pi / 2 \leqslant \theta \leqslant+\pi / 2$, with a concentrated bending moment, $M_{0}$, at the origin, as shown in Fig. $5 a$.

Substituting

$$
u=c_{4} r \log r \cos \theta+c_{5} r \theta \sin \theta
$$

into equations (2), (3) and (4), we have

$$
M_{\theta \theta}=-\frac{D \cos \theta}{r}\left\{(1+v) c_{4}+2 c_{5}\right\}
$$

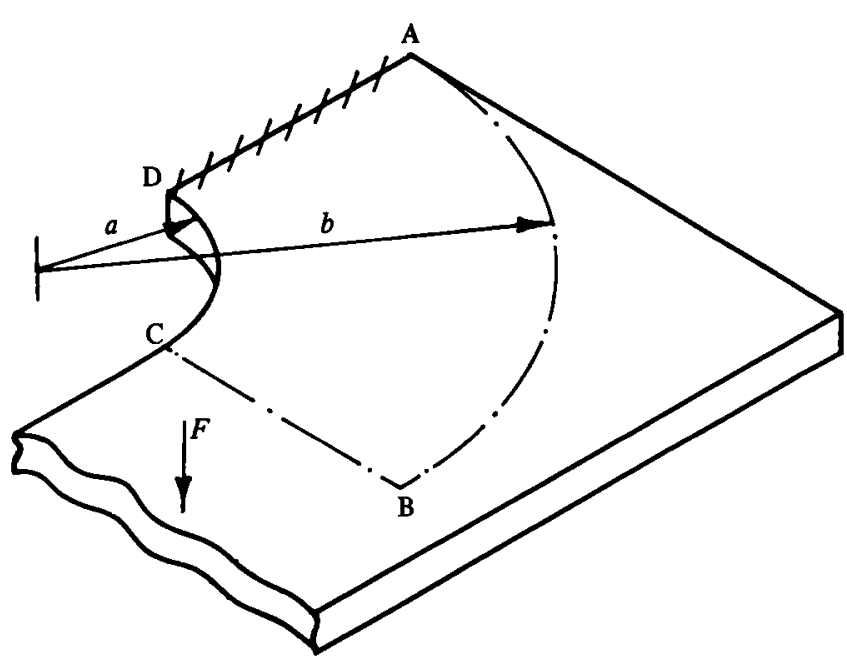

Fig. 4

$$
\left.Q_{\theta}+\frac{\partial M_{r \theta}}{\partial r}=\frac{D \sin \theta}{r^{2}}\left\{(1+v) c_{4}+2 c_{5}\right)\right\}
$$

The edges $\theta= \pm \pi / 2$ will therefore be stress-free if

$$
c_{5}=-(1+v) c_{4} / 2
$$

The value of the remaining constant is then related to $M_{0}$ by considering the equilibrium of a semicircle centred on the origin. The complete solution is

$$
\begin{aligned}
& u=\frac{M_{0}[2 r \log r \cos \theta-(1+v) r \theta \sin \theta]}{(1-v)(3+v) \pi D} \\
& M_{r r}=-\frac{2 M_{0}(1+v) \cos \theta}{(3+v) \pi r} \\
& M_{\theta \theta}=0 \\
& M_{\theta r}=\frac{2 M_{0} \sin \theta}{(3+v) \pi r} \\
& Q_{r}=\frac{2 M_{0} \cos \theta}{(3+v) \pi r^{2}} \\
& Q_{\theta}=\frac{2 M_{0} \sin \theta}{(3+v) \pi r^{2}}
\end{aligned}
$$

It will be observed from equations (4), (27), (28) and (30) that the conditions for the edge $\theta=$ constant are satisfied for all values of $\theta$. Hence, the above solution can be adapted to the problem of a concentrated moment applied at the vertex of a wedge-shaped plate by an appropriate change in the value of $M_{0}$. If the wedge occupies the region $-\varphi \leqslant \theta \leqslant+\varphi$ or $(\pi / 2-\varphi) \leqslant \theta \leqslant$ $(\pi / 2+\varphi)$ (see Fig. 6). then $M_{0}$ in equations (25)-(30) should be replaced by the expression

$$
(3+v) \pi M_{0} /[2(3+v) \varphi-(1-v) \sin 2 \varphi]
$$

In particular, Fig. $6 b$ with $\varphi=\pi / 2$ corresponds to the semi-infinite plate with a concentrated twisting moment 

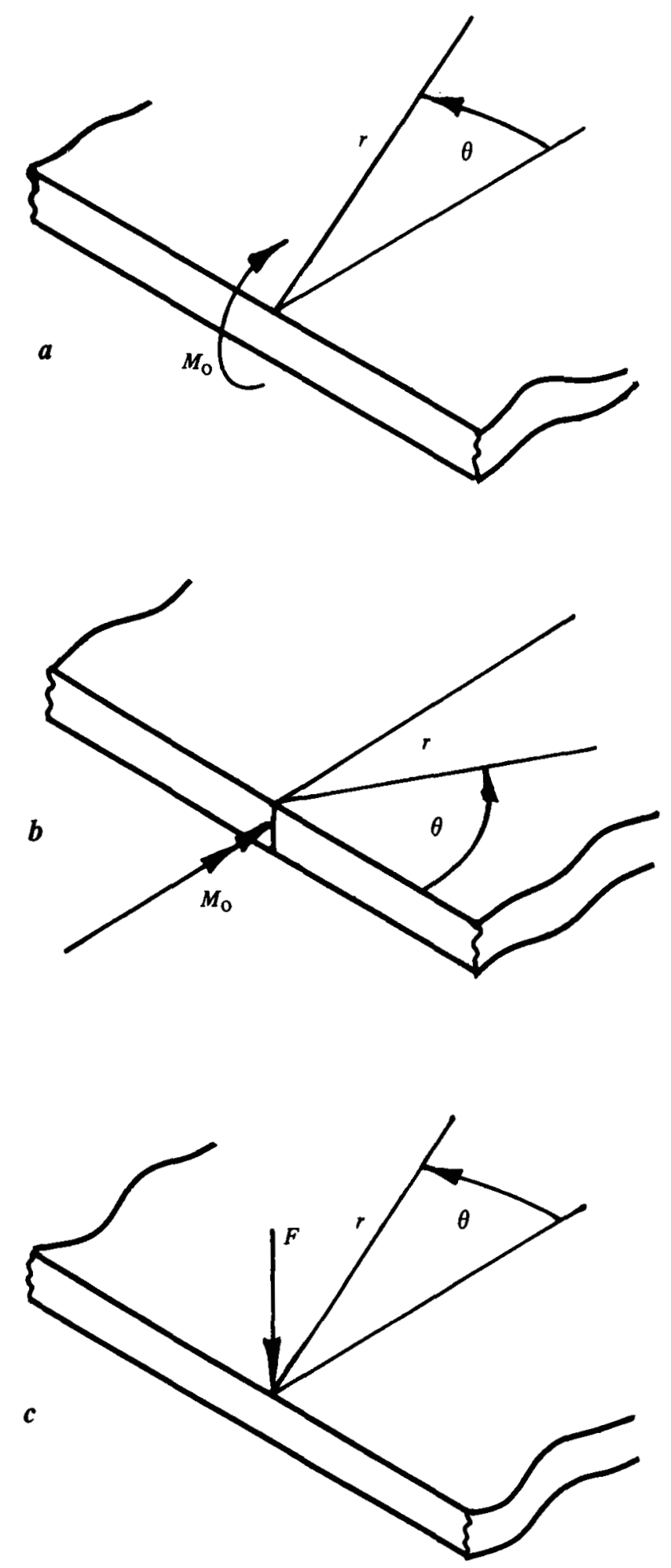

Fig. 5

at the origin (see Fig. $5 b$ ), for which equations (25)-(30) provide the solution. Notice that in each case $\theta$ is measured from the plane of the moment. Alternatively, the displacement field for a twisting moment referred to the co-ordinate system of Fig. $6 a$ is

$$
u=-\frac{M_{0}[2 r \log r \sin \theta+(1+v) \theta \cos \theta]}{(1-v)(3+v) \pi D}
$$

\section{BENDiNG OF A CIRCULAR PLATE BY EDGE MOMENTS}

The similarity between the above results and the stress distribution due to a concentrated in-plane force at the edge of a semi-infinite plate suggests an extension (along the lines of the celebrated paper by Michell (2)) to the problem of a circular plate subjected to a selfequilibrating system of edge moments. This latter problem has been treated by $\mathrm{Yu}$ (3) by complex variable methods, and closed-form solutions were obtained to certain examples.

The solution of equations (25)-(30) is applied to the circular plate $r=2 a \cos \theta$ shown in Fig. 7a. Values of bending and twisting moments $\left(M_{R R}, M_{R \varphi}\right)$ and shear force $\left(Q_{R}\right)$ per unit length are found from the equilibrium of the elemental triangle shown, giving

$$
\begin{aligned}
& M_{R R}=\frac{M_{0}}{2 \pi a}\left\{\frac{(1+v)}{(3+v)}-\cos \varphi\right\} \\
& Q_{R}+\frac{1}{a} \frac{\partial M_{R \varphi}}{\partial \varphi}=\frac{M_{0} \cos \varphi}{2 \pi a^{2}}
\end{aligned}
$$

where $R, \varphi$ is a system of polar co-ordinates whose origin, $\mathrm{O}$, is at the centre of the plate.

The bending moment, $M_{R R}$, can be reduced to zero at the edge $R=a$ by superposing the displacement field:

$$
u=\frac{M_{0} R^{2}}{4 \pi a D(3+v)}\{1-R / a \cos \varphi\}
$$

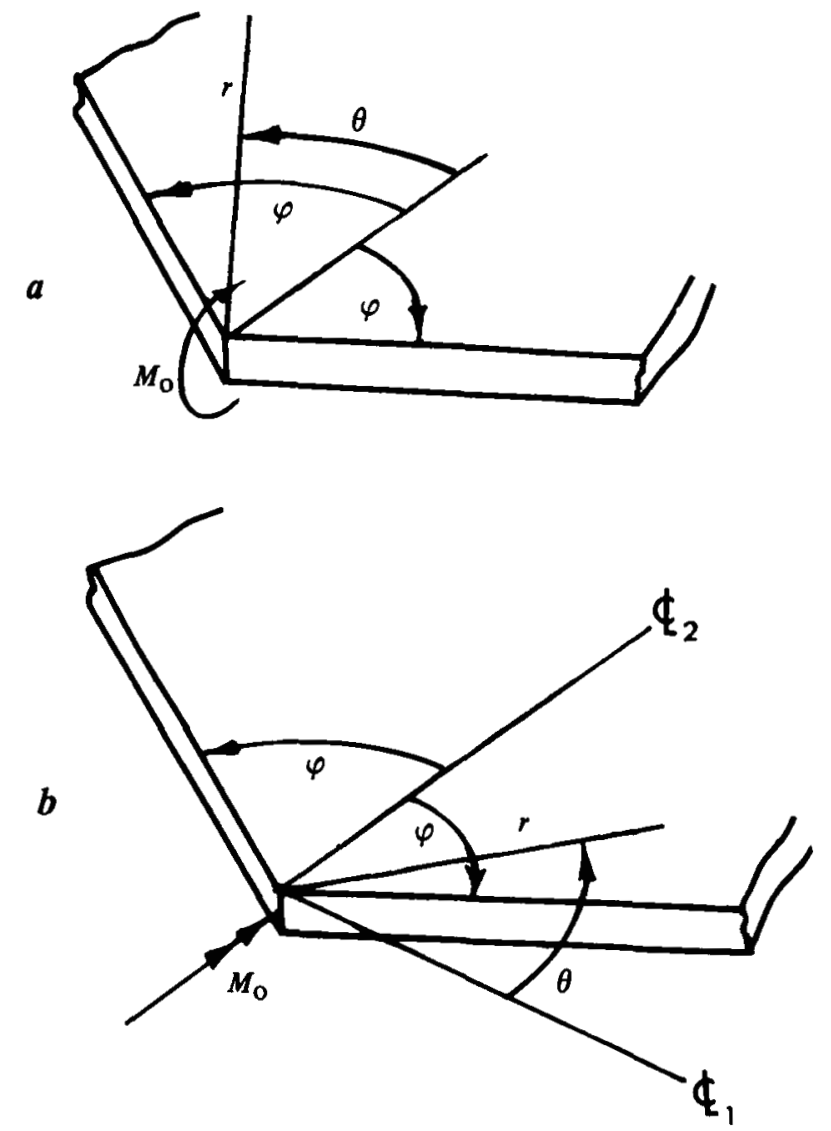

Fig. 6 

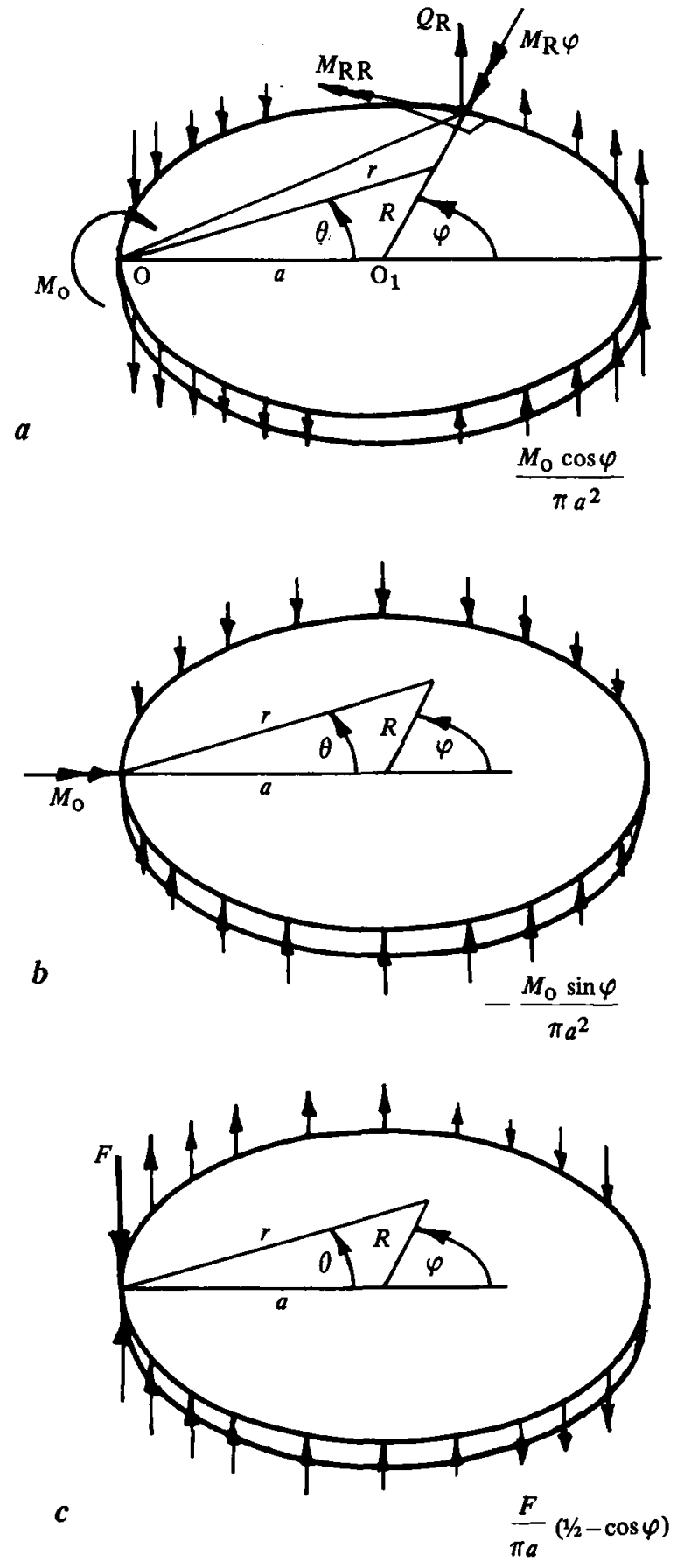

Fig. 7

giving the solution

$$
\begin{aligned}
& u=\frac{M_{0}[2 r \log r \cos \theta-(1+v) r \theta \sin \theta]}{(1-v)(3+v) \pi D} \\
& +\frac{M_{0} R^{2}[1-R / a \cos \varphi]}{4 \pi a D(3+v)}
\end{aligned}
$$

The corresponding solution for a twisting moment applied to the edge at $\varphi=\pi$ (see Fig. 7b) can be obtained by a similar method, and is

$$
\begin{aligned}
& u=-\frac{M_{0}[2 r \log r \sin \theta+(1+v) r \theta \cos \theta]}{(1-v)(3+v) \pi D} \\
& +\frac{M_{0} R^{3} \sin \varphi}{4 \pi a^{2} D(3+v)}
\end{aligned}
$$

Force components are readily found by substitution in equations (2) and (3).

In each case, the only loading on the edge of the plate is the concentrated moment and a balancing transverse force $\left(Q_{R}+1 / a \partial M_{R \varphi} / \partial \varphi\right)$ which varies sinusoidally with $\varphi$. We can therefore write down the solution to the problem of an unloaded circular plate with free edges, subjected to any self-equilibrating system of concentrated edge bending and twisting moments by superposition of equations (35) and (36), since the distributed transverse forces that are then invoked must be selfcancelling. A similar system of self-cancelling support forces has been used for the related problem of the bending and twisting of a circular ring (4).

\section{BENDING OF A PLATE BY A CONCENTRATED TRANSVERSE FORCE AT THE EDGE}

A concentrated twisting moment at the edge of a plate may be regarded as the derivative of a transverse force along that edge. It follows that the solution for a concentrated transverse force can be obtained by a suitable integration of equation (31) for a semi-infinite plate, or equation (36) for a circular plate. Alternatively, Timoshenko's solution (5) for a concentrated force at an interior point of a large plate may be combined with a non-axisymmetric term $\dagger$ of the form $A r^{2}(\theta \sin 2 \theta-\log r$ $\cos 2 \theta$ ) to make a diameter through the origin stress-free.

For the semi-infinite plate $(-\pi / 2 \leqslant \theta \leqslant+\pi / 2)$ subjected to a force, $F$, at the origin (see Fig. $5 c$ ) we obtain

$$
\begin{aligned}
& u=\frac{F r^{2}}{2 \pi(3+v) D}\left\{\frac{(1+v)}{(1-v)}[\theta \sin 2 \theta-\log r \cos 2 \theta]\right. \\
& \left.+\log r+\frac{1}{(1-v)}\right\}
\end{aligned}
$$

The corresponding force components are

$$
\begin{aligned}
& M_{r r}=-\frac{F}{2 \pi(3+v)}\{(1+v)[(2 \log r+3)(1-\cos 2 \theta) \\
& +2 \theta \sin 2 \theta]+2(1-v)\} \\
& M_{\theta \theta}=-\frac{F(1+v)}{2 \pi(3+v)}\{(2 \log r+3)(1+\cos 2 \theta) \\
& -2 \theta \sin 2 \theta\} \\
& M_{r \theta}=-\frac{F(1+v)}{2 \pi(3+v)}\{(2 \log r+3) \sin 2 \theta+2 \theta \cos 2 \theta\} \\
& Q_{r}=-\frac{2 F}{\pi(3+v) r} \\
& Q_{\theta}=0
\end{aligned}
$$

The corresponding solution for a circular plate depends upon the distribution of the support reaction around the edge. Following the method used for edge moments, we support the concentrated force, $F$, by a uniform reaction, $F / 2 \pi a$, and a sinusoidally varying reaction, $-F \cos \varphi / \pi a$ (see Fig. 7c). It then follows that, if a selfequilibrating system of forces and moments is applied to the plate, the support reactions will be self-cancelling.

† Note that functions of the form $r^{n}(\theta \sin n \theta-\log r \cos n \theta)$ are harmonic for all $n$ (including non-integer values). 
The solution is developed by applying to the edge of the plate a twisting moment which increases linearly around the circumference from zero at $\varphi=-\pi$ to $F$ at $\varphi=+\pi$. The discontinuity in $M_{\boldsymbol{R} \varphi}$ at $\varphi= \pm \pi$ is equivalent to a concentrated transverse force, $F$, whilst the linear increase in $M_{R \varphi}$ around the circumference introduces a uniform transverse edge support. The sinusoidally varying term in the support reaction is automatically invoked by using the solution of equation (36).

The integral obtained for the displacement is, unfortunately, not expressible in closed form, but its derivatives are

$$
\begin{aligned}
& \frac{\partial u}{\partial R}=\frac{F}{2 \pi(3+v) D a}\left\{\frac{3 R^{2} \cos \varphi}{2}+R a\right. \\
& \left.-\frac{2(1+v) a^{2} \theta \sin \varphi}{(1-v)}-\frac{a\left[r^{2}+v\left(a^{2}-R^{2}\right)\right] \log r / a}{(1-v) R}\right\} \\
& \frac{\partial u}{\partial \varphi}=\frac{F}{2 \pi(3+v) D a}\left\{-\frac{R^{3} \sin \varphi}{2}\right. \\
& \left.-\frac{2 a^{2} R \log r / a \sin \varphi}{(1-v)}+\frac{2(1+v) a r \theta \cos \theta}{(1-v)}\right\}
\end{aligned}
$$

where

$$
\begin{aligned}
& \theta=\arctan \left\{\frac{R \sin \varphi}{a+R \cos \varphi}\right\} \\
& r=\sqrt{R^{2}+a^{2}+2 a R \cos \varphi}
\end{aligned}
$$

Expressions for the force components in closed form can now be obtained by substituting these results into equations (2) and (3). A series solution to a problem of this type is given by Timoshenko (6).

\section{CONCLUSIONS}

Closed-form solutions have been obtained for the annular plate transmitting a transverse moment and shear force, and for circular and semi-infinite plates subjected to concentrated moments and forces at the edges.

The results can be used to approximate the stress distribution in a loaded plate supported along part of one edge, the discontinuity being relieved by a small fillet radius.

\section{ACKNOWLEDGEMENTS}

The author wishes to thank Messrs Ames Crosta Ltd and Newcastle University Design Unit for permission to publish certain of the above results.

\section{APPENDIX \\ REFERENCES}

(1) MANSFIELD, E. H. The bending and stretching of plates 1964 (Macmillan, New York).

(2) MICHELL, J. H. 'Elementary distributions of plane stress', Proc. London Math. Soc. 1900 32, 35-61.

(3) YU, Y. Y. 'Bending of isotropic thin plates by concentrated edge couples and forces', J. appl. Mech. 1954 21, 129-139.

(4) BARBER, J. R. 'Force and displacement influence functions for the circular ring', J. Strain Analysis 1978 13, 77-81.

(5) TIMOSHENKO, S. P. and WOINOWSKY-KRIEGER, S. Theory of plates and shells 1959, 325, (McGraw-Hill, New York).

(6) ibid. 293. 